\title{
IMPACTO DE LA VARIABILIDAD CLIMÁTICA ESTACIONAL EN LA FENOLOGÍA DE PERA (Pyrus communis L.) CV. WILLIAMS EN RÍO NEGRO-ARGENTINA
}

\section{IMPACT OF SEASONAL CLIMATE VARIABILITY ON THE PHENOLOGY OF PEAR (Pyrus communis L.) CV. WILLIAMS FROM RÍO NEGRO- ARGENTINA}

\author{
Andrea Betiana Rodríguez ${ }^{1 *}$, Angel Rafael Muñoz ${ }^{1}$, Mariela Curetti ${ }^{1}$, María Dolores Raffo ${ }^{1}$ \\ ${ }^{1}$ Instituto Nacional de Tecnología Agropecuaria (INTA), Estación Experimental Agropecuaria Alto \\ Valle, Ruta Nacional 22 km 1190 (CC 782), General Roca, Argentina \\ *Autor para correspondencia E-mail: rodriguez.andrea@inta.gob.ar
}

\section{RESUMEN}

La variabilidad interanual de la fecha de floración en peral Pyrus communis L. influye sobre el rendimiento del cultivo. La temperatura del aire es el principal regulador del desarrollo fenológico en especies frutales de hoja caduca, y provoca la variabilidad interanual de la fecha de floración para el peral. Los adelantos y retrasos mayores a $\mathbf{1 0}$ días generan importantes complicaciones en la calidad y cantidad de producción. El objetivo de este trabajo fue evaluar índices bioclimáticos que permitan prever con anticipación el momento de floración del peral: Para el estudio se trabajó con los registros térmicos de la serie 1971-2016, utilizando los valores de máximas y mínimas diarias y las fechas de plena floración del mismo período. Los índices evaluados responden satisfactoriamente para estimar el momento de plena floración del peral cv. Williams. La mayor probabilidad de ocurrencia de adelantos de plena floración, mayores a 10 días respecto a la media, ocurre con una acumulación óptima de horas de frío y una acumulación de unidades de calor que alcanza el 70\% del requerimiento total antes del 11 de septiembre. Por el contrario, la mayor probabilidad de ocurrencia de floraciones con un retraso mayor a los $\mathbf{1 0}$ días con respecto al valor medio, suceden con un déficit de horas de frío y un déficit en la acumulación de unidades de calor, cubriendo el 50\% del requerimiento de calor total después del 11 de septiembre. Los índices bioclimáticos utilizados se adaptan eficientemente a las condiciones térmicas de la zona de estudio y representan de forma satisfactoria la respuesta ecofisiológica del cultivo.

Palabras clave: endodormancia, ecodormancia, índices bioclimáticos, clima, fenología, pera, Pyrus.

\section{ABSTRACT}

Interannual variability in timing of bloom in pear (Pyrus communis L.) affects crop yield. Air temperature is the main regulator of the phenological development of deciduous fruit trees and causes the interannual variability of flowering date for the pear tree. Bloom occurring $\mathbf{1 0}$ days earlier or later than normal has a high impact on fruit quality and yield. The objective of this work was to evaluate bioclimatic indices that allow predicting the flowering time of pear trees. Temperature records of the 1971-2016 series were analyzed, using daily maximum and minimum values and the full flowering dates of the same period. The assessed indexes allowed for a proper estimation of full flowering date of pear tree cv. Williams. The greatest probability of occurrence of full bloom, earlier

Recibido: 23 septiembre 2019. Aceptado: 27 diciembre 2019. 
10 days than average, occurs with an optimal accumulation of chilling hours and an accumulation of heat units that reaches $70 \%$ of the total requirement before September 11 . On the contrary, the highest probability of occurrence of blooms with a delay greater than 10 days with respect to the average, occurs with a deficit of chilling hours and a deficit in the accumulation of heat units, covering $50 \%$ of the total heat requirement after September 11. The bioclimatic indices used are efficiently adapted to the temperature conditions of the study area and satisfactorily represent the ecophysiological response of pear.

Key words: endodormancy, ecodormancy, bioclimatic predictors, climate, phenology, pear, Pyrus

\section{INTRODUCCIÓN}

En la Patagonia Argentina el cultivo de peras Pyrus communis L. se concentra en los valles irrigados de las provincias de Río Negro y Neuquén. Actualmente la superficie plantada de perales es de 20.800 hectáreas (SENASA, 2017). La variedad Williams representa el $40,4 \%$ de la superficie cultivada con perales en las provincias de Río Negro y Neuquén, seguida por 'Packham's Triumph' (28,8\%), 'Beurre D'Anjou' $(14,1 \%)$ y 'Abate Fetel' (5,3\%) entre las más importantes (SENASA, 2017). En el Alto Valle de Rio Negro desde el año 1971 se registra la fecha de plena floración y autorización de cosecha para la variedad Williams (Benítez et al., 2005).

El momento de plena floración en pera 'Williams' marca el comienzo del ciclo de crecimiento y la cronología de una serie de prácticas culturales y respuestas ecofisiológicas que condicionan el rendimiento en términos de calidad y cantidad (Rodríguez et al., 2011). La pera 'Williams' es un cultivo de ciclo corto y de rápido crecimiento siendo el tamaño y el estado de madurez de los frutos dos de los principales parámetros de calidad comercial. La variabilidad de la fecha de plena floración tiene efectos directos sobre el crecimiento del fruto; cuanto más tardía sea menor edad tendrá el fruto al momento en que se desencadenen los procesos asociados a la madurez, por lo cual su tamaño estará por debajo de los parámetros comerciales establecidos (Rodríguez, 2011). Por otro lado, con adelantos de plena floración cercanos a los 10 días con respecto a la fecha media, el tamaño del fruto a cosecha puede ser favorecido por tener mayor edad; sin embargo, el cultivo está expuesto a una mayor probabilidad de daño por heladas tardías y la posibilidad de periodos térmicamente menos favorables durante la fecundación y fase de división celular del fruto (Rodríguez et al., 2011).

De acuerdo a lo observado en diferentes ciclos de crecimiento, existe una gran variabilidad interanual en las fechas de floración (desde el 16 de septiembre al 07 de octubre) (Rodríguez y Muñoz, 2006). Los índices agro-meteorológicos son expresiones cuantitativas de la relación existente entre las condiciones meteorológicas y el comportamiento del cultivo. Identificar y desarrollar parámetros locales de estos índices es fundamental para entender escenarios productivos actuales y prospectivos asociados al cambio climático (Pascale y Damario, 2004).

La longitud del día y la temperatura del aire regulan el proceso de aclimatación de la planta a las bajas temperaturas, sucediendo un aumento gradual de la tolerancia al frío a partir del otoño (Horvath, 2009). Durante la segunda mitad del invierno, la resistencia al frío disminuye gradualmente, este proceso es denominado desaclimatación. La temperatura del aire ejerce un papel fundamental en la tasa a la cual los órganos invernantes pierden su resistencia a las bajas temperaturas; la desaclimatación es acompañada por rehidratación y disminución de los niveles de azúcares (Szalay et al., 2010; Pagter et al., 2011). La desaclimatación y la ecodormancia ocurren en paralelo (Welling y Palva, 2006). En la fase de ecodormancia, la tasa de crecimiento se incrementa con el aumento de la temperatura (Lang et al., 1987; Luedeling et al., 2013).

Las horas de frío constituyen el índice agrometeorológico utilizado tradicionalmente para cuantificar la duración de la fase de endodormancia, y expresa la cantidad de horas acumuladas con temperaturas del aire menores o iguales a $7^{\circ} \mathrm{C}$. Si bien otros modelos consideran como efectivas temperaturas de $2,5^{\circ} \mathrm{C}$ a $9,1^{\circ} \mathrm{C}$, la temperatura base de $7^{\circ} \mathrm{C}$ se ajusta con el comportamiento fenológico del peral (Rodriguez et al., 2011). La fase de ecodormancia es consecutiva a la endodormancia y se cuantifica a través de un modelo de acumulación térmica, restándole a la temperatura media diaria un umbral de $7^{\circ} \mathrm{C}$ para frutales de pepita (Pascale y Damario, 2004; Campoy, 2011). Localmente se han estimado los umbrales de referencia en 1000 horas de frío para completar la fase de endodormancia, y 180 unidades de calor, para llegar al estado fenológico de plena floración (Rodríguez, 2011).

Los periodos de entrada y salida de la endodormancia de la yema están acompañados de aumentos en los niveles de inhibidores hasta 
el estado pleno de reposo (endodormancia) seguido por una progresiva disminución de los mismos y un aumento paulatino de promotores de crecimiento (Westwood, 1982; Druart et al., 2007). En los frutales el proceso de aclimatación al frío tarda de semanas a meses, completándose a mitad del invierno; en cambio el proceso de desaclimatación es más rápido y puede ocurrir en pocos días o semanas (Kalberer et al., 2006). De acuerdo a Kalberer et al. (2006), cuando ocurre el incremento térmico antes de que se cumpla el requerimiento en frío invernal, se produce un aumento del consumo de sustancias de reservas y azúcares solubles para cubrir los requerimientos energéticos de la respiración. Esto provoca una desaclimatización llamada pasiva, que deja a la planta más susceptible a la desaclimatización activa (Szalay et al., 2010). Por esta razón, la respuesta de los frutales a la variabilidad térmica interanual es muy compleja; aun así las horas de frío y las unidades de calor pueden ser índices bioclimáticos que nos permiten tener una aproximación del comportamiento fenológico de los frutales (Pascale y Damario, 2004; Campoy et al., 2011).

Los objetivos de este trabajo fueron: 1) analizar la variabilidad de las fechas de plena floración de pera cv. Williams; 2) evaluar la variabilidad de las temperaturas durante el periodo de endodormancia y ecodormancia a través de los índices bioclimáticos: horas de frío (HF) y unidades de calor (UC); y 3) determinar la relación existente entre los índices bioclimáticos y las fechas de plena floración de peras cv. Williams.

\section{MATERIALES Y MÉTODOS}

\section{Ubicación de estación automática}

Se trabajó con los registros térmicos anuales de la serie 1971-2016, utilizando los valores de máximas y mínimas diarias y las fechas de plena floración del mismo período. Las variables climáticas analizadas pertenecen a los registros de las estaciones meteorológicas de la Estación Experimental Alto Valle del Instituto Nacional de Tecnología Agropecuaria (INTA EEA Alto Valle) (3901 Sur; 67으 6 Oeste; $242 \mathrm{msnm}$ ).

\section{Registros fenológicos}

Las observaciones fenológicas se realizaron sobre parcelas experimentales de peral 'Williams' ubicadas en la Estación Experimental Alto Valle del INTA. Se utilizaron registros fenológicos históricos desde 1971 a 2004 y se continuaron hasta el 2016 utilizando la metodología original (Fleckinger, 1965) (Fig. 1).

El análisis interanual de las fechas de plena floración se realizó a través del análisis de

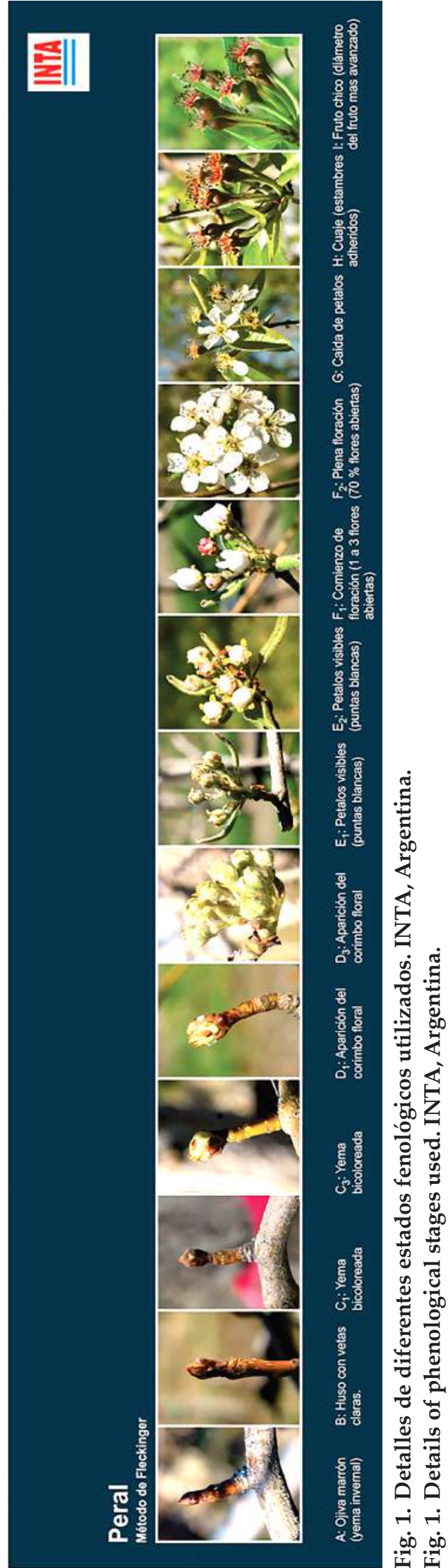


anomalías con respecto a la fecha media de plena floración. A partir de esta información se determinaron las fechas extremas de plena floración y se evaluó su variabilidad analizando periodos de diez años (frecuencia relativa decadial).

\section{Cálculo y análisis de índices bioclimáticos}

Los índices bioclimáticos utilizados fueron horas de frío (HF) y unidades de calor (UC), calculados para cada año de la serie 1971-2016. El cálculo de horas de frío se realizó contabilizando las horas con temperaturas del aire menores o iguales a $7^{\circ} \mathrm{C}$ (temperatura base) durante los meses de mayo a agosto. La variabilidad interanual de HF se analizó a través del cálculo de anomalías; es decir la diferencia en días del momento en que se cumple con el requerimiento (umbral regional $1000 \mathrm{HF}$ ) con respecto a la fecha media de la serie analizada (Rodríguez, A., 2011). Posteriormente se categorizaron como inviernos óptimos (IO) aquellos años en donde el requerimiento en frío se cubre antes de la fecha media, e inviernos con déficit (ID) cuando el requerimiento se cumple después de la fecha media o no se cubre.

Las unidades de calor fueron calculadas a través de un modelo de acumulación térmica, restándole a la temperatura media diaria un umbral de $7^{\circ} \mathrm{C}$. Para el análisis de variabilidad interanual se utilizó como parámetro el umbral de $180 \mathrm{UC}$, requerimiento calculado regionalmente (Rodríguez, A., 2011). Se evaluó a través de percentiles la fecha más frecuente en la que se cumple el 50 y $70 \%$ del requerimiento. Con esas fechas se identificaron eventos extremos (días de adelanto o retraso en el cumplimiento del requerimiento). Posteriormente se categorizó la fase de ecodormancia en función del momento en que se cubre el $50 \%$ y el $70 \%$ del requerimiento de calor con respecto al valor del percentil 75; en primavera normal (PN) cuando el 50\% y $70 \%$ del requerimiento calórico se cumple en la fecha correspondiente al percentil 75 de la serie respectivamente ( PN50 y PN70); primavera fría (PF) cuando el 50\% del requerimiento se cubre después del percentil 75 (valor expresado como día juliano) ; y primavera cálida (PC) cuando el $70 \%$ del requerimiento se cubre antes de la fecha del percentil 75 para el acumulado del 50\%.

\section{Procedimiento estadístico}

Se realizó un análisis estadístico descriptivo para las fechas en que se alcanza el requerimiento de frío del cultivar en estudio, determinando valores medios, y percentiles $10,25,50,75,90$. Para el análisis de variabilidad se determinaron anomalías sobre valores acumulados mensuales de horas de frío. Posteriormente se realizó un análisis de regresión lineal entre las fechas en que se alcanza el requerimiento en frío y las anomalías para cada mes. Se consideró y analizó al período de ecodormancia desde que se alcanza el requerimiento de frío hasta el estado de plena floración. Para evaluar la variabilidad térmica de la fase post-reposo se analizaron los valores de moda, media y percentiles de la fecha en que se acumula un $50 \%$ y un $70 \%$ del requerimiento de calor medio. Para el estudio de variabilidad en fechas de plena floración se estimaron probabilidades de ocurrencia a través de un análisis de frecuencias. Todas las fechas fueron trabajadas a través de una escala continua de tiempo, días julianos (DJ). Es decir cada fecha se traduce como un número correspondiente a la acumulación continua de días solares, cuyo origen es el día 1 de enero. En cada caso el análisis estadístico fue hecho con el programa InfoStat (2009).

\section{RESULTADOS Y DISCUSIÓN}

\section{Variabilidad interanual de fechas de plena floración}

$\mathrm{Al}$ analizar las anomalías (Fig. 2) se observa una variabilidad media de \pm 5 días con respecto al 25 de septiembre (plena floración del 20 al 30 de septiembre) y fechas extremas con desplazamientos mayores o iguales a 10 días (plena floración del 15 al 18 de septiembre y del 2 al 9 de octubre). La frecuencia de ocurrencia de fechas extremas de plena floración es del 11,4\% (2 en 20 años) para el adelanto de la floración, y de 13,6\% (3 en 20 años) para el retraso de la floración.

Las floraciones tardías, ocurridas durante los primeros días de octubre fueron más frecuentes desde la década de los 90. Mediante un análisis decadial, se observó que existe una tendencia de aumento de retrasos extremos en las fechas de plena floración (Fig. 3). La variabilidad climática interanual ha sido muy marcada en los últimos 6 años. Las fechas extremas de los últimos 45 años se registraron en el 2014 (15 de septiembre) y en el 2015 (9 de octubre). En el primer caso se observó que el fruto aceleró su proceso de madurez por tener edad suficiente, mayor a 110 días (Gomila, 2015); en el segundo caso el tamaño del fruto fue seriamente afectado porque la duración de su ciclo de crecimiento disminuyó (Candan et al., 2016).

\section{Variabilidad interanual de índices bioclimáticos:}

El análisis de índices bioclimáticos, como horas de frío y unidades de calor, es una herramienta útil para lograr cuantificar el complejo proceso de desarrollo de los órganos reproductivos en especies frutales, ligado principalmente al 


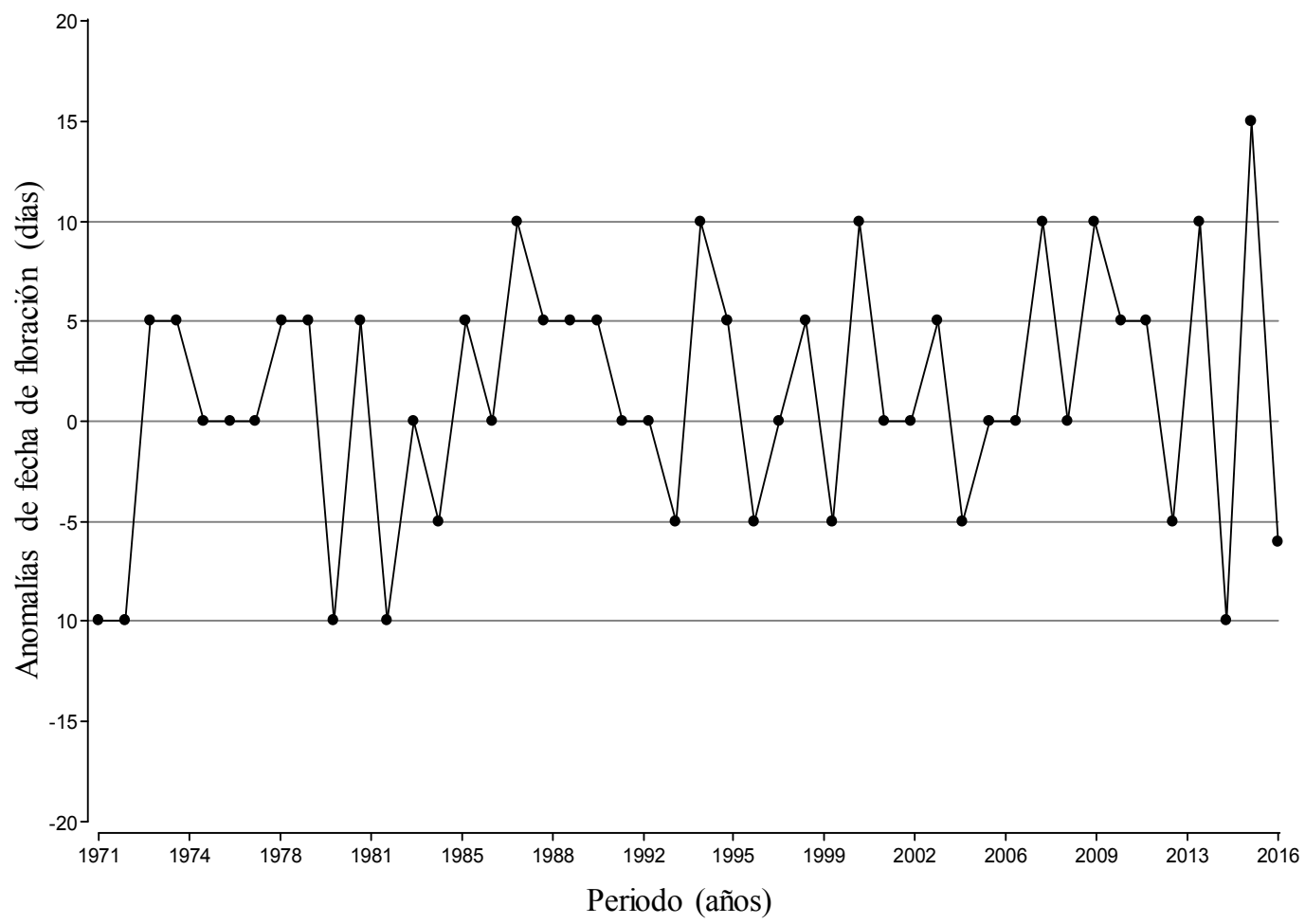

Fig. 2. Anomalías de fechas de plena floración con respecto a la fecha media para pera cv. Williams. 25 septiembre.

Fig. 2. Variability in timing of full bloom with respect to the average date for pear cv. Williams. September 25.

balance hormonal y distribución de carbohidratos de reserva. Conocer el comportamiento térmico pre-floración de los últimos 45 años, nos permite predecir el momento de plena floración y puede darnos una aproximación acerca de lo que ocurre durante el desarrollo de las yemas florales.

El $90 \%$ de los años estudiados cumplen con el requerimiento de frío para pera $\mathrm{cv}$. Williams antes del 8 de agosto (Fig. 4) (percentil 90, DJ 221). La fecha media de acumulación de 1000 horas frío es el 26 de julio (DJ 208). En 8 de cada 10 años el requerimiento se cumple entre el 16 de julio y 1 de agosto. En la Fig. 4 se observa la variabilidad interanual de las fechas en que se cumple el requerimiento de frío, expresado como anomalía con respecto al valor medio (DJ 208, 26 julio), en dónde la dispersión no es mayor a los 20 días. Para las situaciones extremas se observa que: a) dos de cada 10 años alcanzan el requerimiento en frío de la variedad al menos 10 días antes con respecto al valor medio, estas condiciones predisponen a los frutales a tener floración anticipada; b) los retrasos mayores o iguales a 10 días también tienen una ocurrencia de 2 cada 10 años afectando directamente la heterogeneidad en el desarrollo de las yemas florales al momento del estado fenológico C (métodología Fleckinger,
1965), interpretado comúnmente como falta de frío. Por otra parte, adelantos y retrasos de 5 a 10 días se dan con una menor frecuencia 1 cada 10 años.

Del análisis de regresión lineal entre la variabilidad del momento en que se alcanza el requerimiento en frío y la variabilidad térmica de los meses de mayo a julio, se observa que el mes de junio explica un $63 \%$ de esta variabilidad, julio un $42 \%$ y mayo $35 \%$ (Tabla 1). En estos términos podemos decir que las temperaturas del mes de junio tienen la mayor incidencia sobre la acumulación de frío de pera cv. Williams y sobre su impacto en el desarrollo fenológico.

El tiempo en días para llegar al estado de plena floración varía principalmente en función de las condiciones térmicas durante el reposo y post-reposo (Campoy et al., 2011). De acuerdo a estudios anteriores se estimó que el requerimiento calórico post-reposo, necesario para llegar al estado de plena floración, es de $180 \pm 23$ unidades de calor (Rodríguez, 2011). Al evaluar la variabilidad térmica de la fase postreposo se observó que el 11 de septiembre es una fecha media clave para poder inferir el desarrollo fenológico del peral (Fig. 5). En el 75\% de los años analizados se acumula el $50 \%$ de las unidades de 


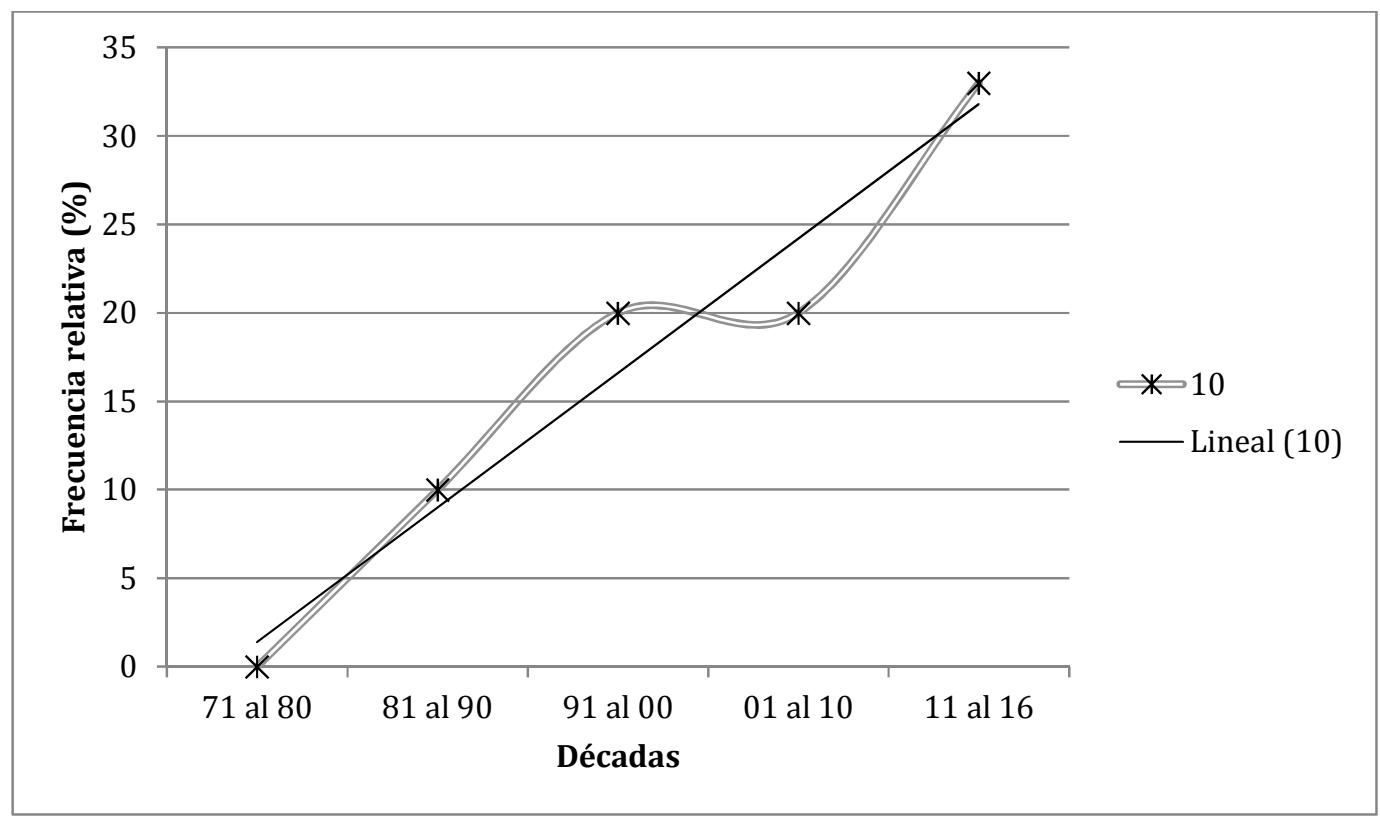

Fig. 3. Frecuencia relativa decadial de retrasos extremos (10 días) de las fechas de plena floración. Línea de tendencia en base a un modelo de regresión lineal $\left(\mathrm{r}^{2} 0,94\right)$.

Fig. 3. Relative frequency per decade of late bloom (10 days). Trend line based on a linear regression model ( $\left.r^{2} 0.94\right)$.

calor requeridas al 11 de septiembre. En función de esto, se consideró estar ante un período post reposo frío cuando se alcanza dicho valor después del 11 de septiembre. El percentil 75 para alcanzar el $70 \%$ de las unidades de calor requeridas es el 18 de septiembre, analizar la anomalía del 70\% UC con respecto al 11 de septiembre permite identificar condiciones extremas de periodos post reposo cálidos.

La variabilidad térmica entre los diez a quince días inmediatos a la finalización del requerimiento en frío es muy notable (Fig. 5). Las anomalías negativas muestran que los años extremos alcanzan un $70 \%$ del requerimiento calórico al 11 de septiembre. La frecuencia de estos años es de 3 en 20 años y no se identifica un patrón de ocurrencia. Del mismo modo al analizar la acumulación del $50 \%$ de requerimiento calórico después del 18 de septiembre (anomalías positivas mayores a 7 días), se observó años extremadamente fríos, cuya frecuencia es menor, de 1 en 20 años.

\section{Relación existente entre los índices bioclimáticos y las fechas de plena floración:}

Las distintas especies y variedades difieren tanto en requerimiento de frío durante el invierno, como en necesidades de calor en la época que antecede a la fase fenológica de floración; la fecha de floración de una variedad en un año y localidad determinada depende de cómo y en qué medida se cumplen estos requerimientos térmicos (Citadin et al., 2001; Campoy et al., 2011; Russell et al., 2014; Chmielewski y Götz, 2017). Se ha demostrado que después de cumplido el reposo invernal las yemas pueden permanecer cerradas exclusivamente por factores climáticos; la velocidad en el cambio de fases está regulada principalmente por la temperatura que juega un rol fundamental sobre la síntesis de hormonas de crecimiento (Drepper et al., 2020).

En el presente estudio se realizó un análisis de frecuencias relativas de las fechas de plena floración y de la variabilidad de los índices bioclimáticos analizados, según la categorización: inviernos óptimos (IO) aquellos años en donde el requerimiento en frío se cubre antes del 26 de julio, e inviernos con déficit (ID) cuando el requerimiento se cumple después del 26 de julio o no se cubre. Por otro lado, el comportamiento térmico de la fase de ecodormancia se categoriza en función del momento en que se cubre el $50 \%$ y el $70 \%$ del requerimiento de calor como: primavera normal (PN) cuando el $50 \%$ del requerimiento calórico se cubre al 11 de septiembre (PN50) y cuando el $70 \%$ se alcanza al 18 de septiembre (PN70); primavera fría (PF) cuando el $50 \%$ se cubre después del 11 de septiembre; y primavera cálida (PC) cuando el 70\% del requerimiento se cubre antes del 11 de septiembre. De acuerdo al análisis de variabilidad en las fechas de plena floración se trabajó con 5 sub-periodos en función 
Tabla 1. Número de años analizados $(\mathrm{N})$, coeficiente de regresión $\left(\mathrm{R}^{2}\right)$ y p-valor, del análisis de regresión entre la variabilidad del momento en que se alcanza el requerimiento en frío y la variabilidad térmica de los meses de mayo, junio y julio.

Table 1. Number of years analyzed $(N)$, regression coefficient $\left(R^{2}\right)$ and p-value of the regression analysis between variability of timing when cold requirement is reached and temperature variability in May, June and July.

\begin{tabular}{llll}
\hline Mes & $\mathbf{N}$ & $\mathbf{R}^{\mathbf{2}}$ & p-valor \\
\hline Mayo & 43 & 0,35 & $<0,0001$ \\
Junio & 43 & 0,63 & $<0,0001$ \\
Julio & 43 & 0,42 & $<0,0001$ \\
\hline
\end{tabular}

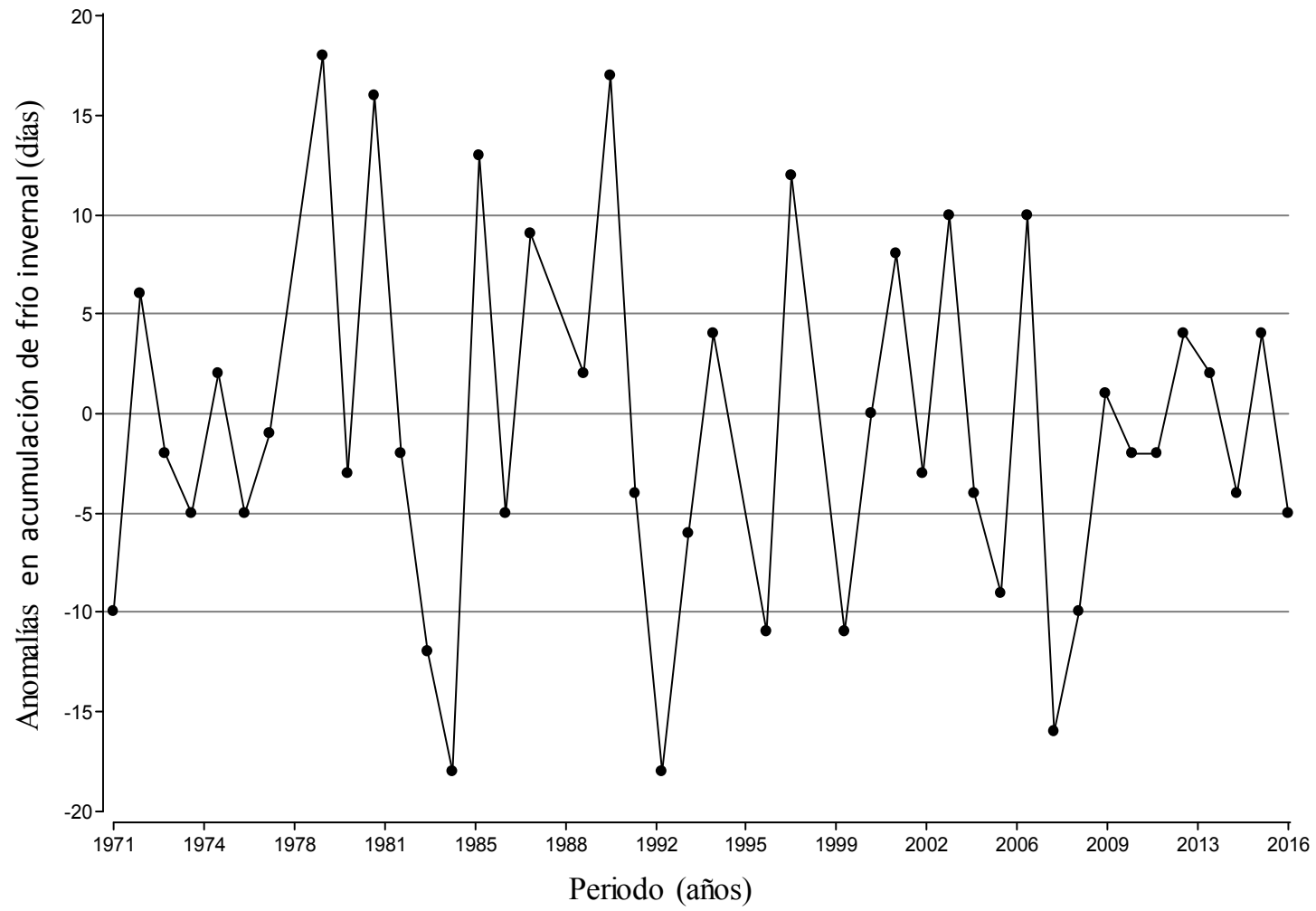

Fig. 4. Variabilidad de acumulación de frío serie 1971-2016, expresada como anomalías en días, de la fecha en que se alcanza el requerimiento de pera cv. Williams. Valores negativos indican adelantos con respecto a la fecha media ( 26 de julio) y valores positivos indican retrasos con respecto a la misma.

Fig. 4. Variability of chilling accumulation for series 1971-2016, in number of days, from the date when the pear cv. Williams requirement is reached. Negative values indicate advance, while positive values indicate delays with respect to the average date (July 26).

de la anomalía al 25 de septiembre (Tabla 2).

Diversos estudios han relacionado el aumento de las temperaturas con el adelanto fenológico en frutales de clima templado (Chmielewski et al., 2004; Wolfe et al., 2005). Analizar la respuesta fenológica de la pera a través del comportamiento conjunto de horas de frío y unidades de calor post reposo, otorga mayor posibilidad de estimación anticipada de la fecha de floración a nivel local.
A través del análisis de frecuencias se realizó una tabla de probabilidades (Tabla 3) combinando los índices bioclimáticos y los momentos de floración sintetizando los periodos probables de plena floración en 3 categorías: temprana, normal y tardía. El análisis indica un $70 \%$ de probabilidad de tener una floración temprana cuando el invierno es óptimo y la primavera cálida. Por otro lado, existe un $83,3 \%$ de probabilidad de tener 


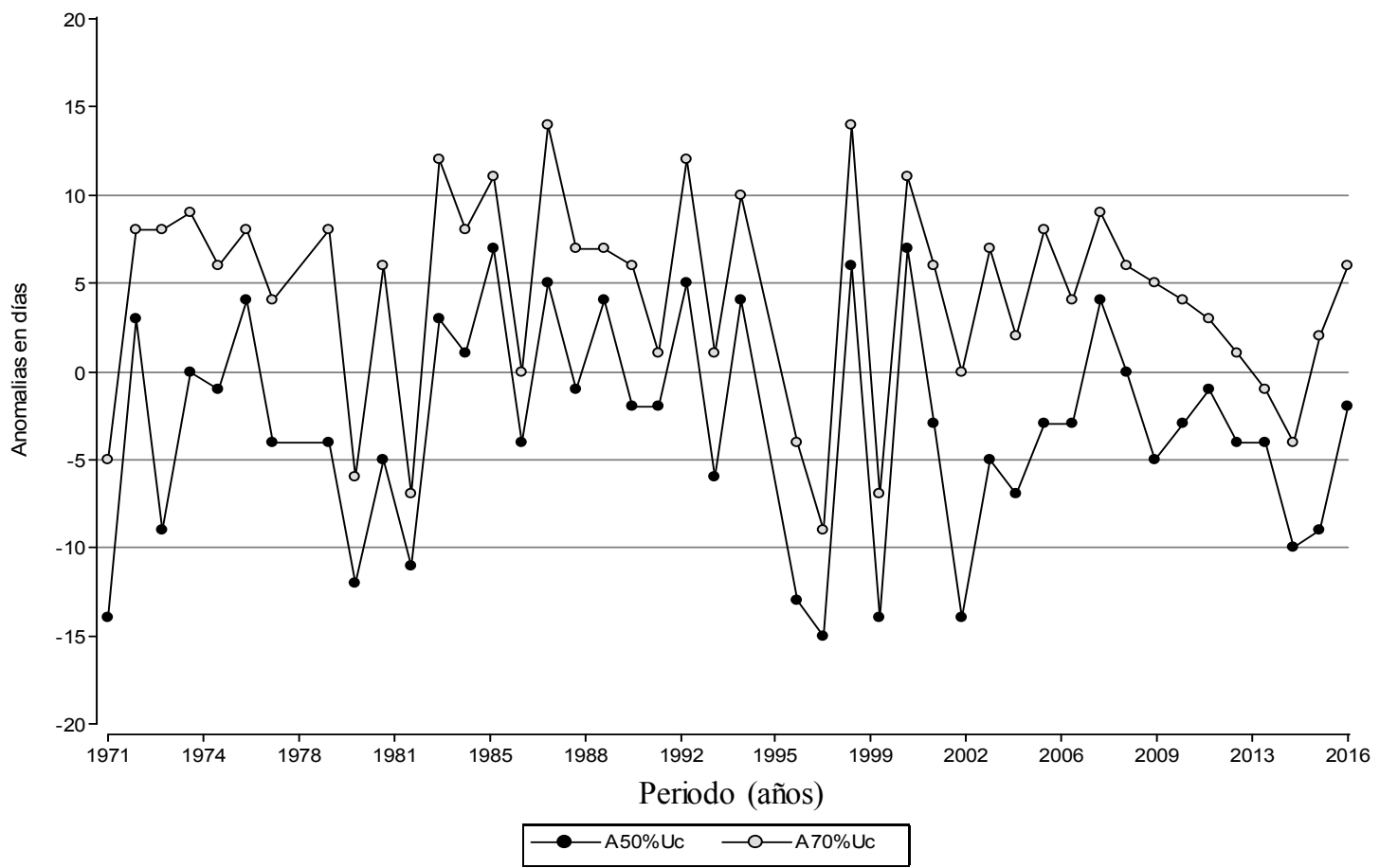

Fig. 5. Variabilidad de los momentos en que se alcanza el 50 y $70 \%$ del total de unidades de calor requeridas para pera cv. Williams (serie 1971-2016), expresada como anomalías en días con respecto al 11 de septiembre. Valores negativos indican fechas anteriores al 11 de septiembre y valores positivos indican fechas posteriores.

Fig. 5. Variability of timing when 50 and $70 \%$ of heat requirement for pear $\mathrm{cv}$. Williams (series 19712016) is reached, expressed as anomalies in days with respect to September 11. Negative values indicate dates before September 11, and positive values indicate dates later September 11.

Tabla 2. Frecuencia relativa de plena floración y valores de anomalías expresadas en días para cada sub-periodo planteado.

Table 2. Relative frequency of full bloom and anomaly values expressed in days for each sub period.

\begin{tabular}{lccccc}
\hline $\begin{array}{l}\text { Momento de } \\
\text { floración }\end{array}$ & $\begin{array}{c}\text { Antes del } \\
\text { 23 septiembre }\end{array}$ & $\begin{array}{c}\text { 20 al 23 de } \\
\text { septiembre }\end{array}$ & $\begin{array}{c}\text { 24 al 26 de } \\
\text { septiembre }\end{array}$ & $\begin{array}{c}\text { Del 27 septiembre } \\
\text { al 1 octubre }\end{array}$ & $\begin{array}{c}\text { Después del } \\
\text { 2 de octubre }\end{array}$ \\
\hline Anomalía & -10 & -5 & 0 & 5 & 10 \\
\hline $\begin{array}{l}\text { Frecuencia relativa } \\
(1971-2016)\end{array}$ & 0,114 & 0,136 & 0,295 & 0,318 & 0,136 \\
\hline
\end{tabular}

una floración tardía con un invierno deficitario y una primavera fría. Las floraciones tardías se dan con mayor probabilidad cuando el invierno es deficitario $(58,3 \%)$.

Sin embargo, también se observó que para una floración normal el requerimiento de frío se alcanzó al 26 de julio, y el requerimiento de calor se cubre paulatinamente en un $50 \%$ al 11 de septiembre, y el $70 \%$ al 18 de septiembre. La ocurrencia de la floración entre el 20 y 23 de septiembre sucede cuando tenemos un invierno óptimo y primaveras normales al 11 de septiembre. Si la floración ocurre entre el 27 de septiembre y 1 de octubre, la acumulación de frío fue deficiente pero el $70 \%$ del requerimiento de calor se alcanzó al 18 de septiembre.

Para las situaciones de fechas extremas de floración se observó que un adelanto mayor o igual a 10 días (antes del 20 de septiembre), ocurre con una acumulación de frío óptima y con una rápida acumulación de calor durante la fase de ecodormancia, alcanzando el $70 \%$ del 
Tabla 3. Probabilidad de adelanto o retraso de plena floración de pera 'Williams' en función del momento en que se cumple el requerimiento de frío y el requerimiento de calor. Categorización de inviernos óptimos y con déficit; categorización de primaveras cálidas (70\% UC antes del 11 de septiembre), normales $(50 \%$ de UC al 11 de septiembre y $70 \%$ al 18 de septiembre) y frías ( $50 \%$ UC después del 11 de septiembre); plena floración temprana (hasta el 23 de septiembre); normal (desde el 24 al 26 de septiembre) y tardía (después del 27 de septiembre).

Table 3. Probability of advance or delay of full bloom date of pear 'Williams' depending on when the requirement of cold and heat are reached. Optimum winter; winter with deficit; warm spring (70\% UC before September 11); normal spring ( $50 \%$ UC on September $11 ; 70 \%$ UC to September 18); cold spring (50\% UC after September 11); early full bloom (until September 23); normal (from September 24 to 26) and late full bloom (after September 27).

\begin{tabular}{llcccc}
\hline $\begin{array}{l}\text { Categoría } \\
\text { de invierno }\end{array}$ & $\begin{array}{c}\text { Categoría de } \\
\text { primavera }\end{array}$ & $\begin{array}{c}\text { Floración } \\
\text { temprana }\end{array}$ & $\begin{array}{c}\text { Floración } \\
\text { normal }\end{array}$ & $\begin{array}{c}\text { Floración } \\
\text { tardía }\end{array}$ & $\begin{array}{c}\text { Frecuencia } \\
\text { relativa }\end{array}$ \\
\hline Optimo & Fría & 0,000 & 0,067 & 0,044 & $\mathbf{0 , 1 1 1}$ \\
& Normal & 0,067 & 0,067 & 0,133 & $\mathbf{0 , 2 6 7}$ \\
& Cálida & 0,156 & 0,067 & 0,000 & $\mathbf{0 , 2 2 2}$ \\
Déficit & Fría & 0,022 & 0,000 & 0,111 & $\mathbf{0 , 1 3 3}$ \\
& Normal & 0,000 & 0,089 & 0,111 & $\mathbf{0 , 2 0 0}$ \\
& Cálida & 0,022 & 0,022 & 0,022 & $\mathbf{0 , 0 6 7}$ \\
Frecuencia relativa & & $\mathbf{0 , 2 6 7}$ & $\mathbf{0 , 3 1 1}$ & $\mathbf{0 , 4 2 2}$ & $\mathbf{1 , 0 0 0}$ \\
\hline
\end{tabular}

UC: unidades calor

requerimiento al 11 de septiembre. Un retraso mayor o igual a 10 días (fechas de floración después del 2 de octubre), está condicionado principalmente por no alcanzar a cubrir el requerimiento de frío al 26 de julio, y tener periodos fríos hasta el 11 de septiembre durante la fase de ecodormancia. En concordancia con Campoy et al. (2011), el rompimiento de la dormancia es progresivo y es resultado de diferentes combinaciones de acumulación de horas de frío y unidades de calor. Cuando se acumulan las unidades de frío mínimas (que permiten el desarrollo fenológico), las necesidades de calor decrecen progresivamente.

A pesar de que algunos modelos combinados (Horas frío-acumulación de calor) son capaces de predecir las fechas de floración dentro de unos pocos días, en relación a la fecha real no todos explican la variación real (Luedeling, 2012). La interacción entre el fotoperíodo y las temperaturas ha sido asociada con el proceso de dormancia en plantas leñosas (Heide, 1974; 2008; Tanino et al., 2010) y su incorporación a los modelos de acumulación de horas frío podría ser de utilidad. Comprender cómo la duración y la temperatura de los días modifican la expresión de genes específicos podría proporcionar información sobre los mecanismos a través de los cuales estos factores ambientales regulan la dormancia (Horvath, 2009).

\section{CONCLUSIONES}

Existe variabilidad interanual en las fechas de floración de pera cv. Williams y los retrasos iguales o superiores a 10 días han aumentado en las últimas décadas.

Se observó variabilidad en la acumulación de horas de frío. Sin embargo, en el $90 \%$ de los años analizados se alcanzó el requerimiento de frío de la variedad antes del 8 de agosto. Las temperaturas del mes de junio tienen la mayor incidencia sobre la acumulación de frío de pera $\mathrm{cv}$. Williams

Las temperaturas pos reposo invernal, ocurridas hasta el 11 de septiembre, explican significativamente el desarrollo fenológico de la pera cv. Williams. Pero el momento de plena floración queda determinado por el comportamiento conjunto de los dos índices bioclimáticos analizados.

El análisis de índices bioclimáticos, como horas de frío (HF) y unidades de calor (UC), reflejan la variabilidad térmica durante los procesos de endodormancia y ecodormancia, y son una herramienta útil para cuantificar el complejo proceso de desarrollo de los órganos productivos. Las combinaciones de los diferentes comportamientos térmicos de los dos índices utilizados, dan como resultado la variabilidad interanual del desarrollo fenológico, y finalmente 
del momento de plena floración.

La fecha media de acumulación del requerimiento de frío y la fecha en la que se alcanza el $50 \%$ del requerimiento de calor resultaron ser parámetros adecuados para estimar el momento de plena floración de pera 'Williams'.

\section{LITERATURA CITADA}

Benítez C., H. Castro, A. Ricca, y S. Vaudagna. 2005. Peras y manzanas: Factores que afectan la calidad de los frutos. 396 p. Ediciones INTA, Buenos Aires, Argentina.

Candan A.P., G. Calvo, y T. Gomila. 2016. Boletín de Madurez. № 2. Temporada 2015-2016. Disponible en http://inta.gob.ar/documentos/ boletin-de-madurez (Consulta 5 mayo de 2017).

Campoy J.A., D. Ruiz, and J. Egea. 2011. Dormancy in temperate fruit trees in a global warming context: A review. Scientia Horticulturae 130(2):357-372.

Citadin I., M. Raseira, F. Herter, and B. Da Silva. 2001. Heat requirement for blooming and leafing in peach. HortScience 36(2):305-307.

Chmielewski, F.M., A. Müller, and E. Bruns. 2004. Climate change and trends in phenology of fruits trees and field crops in Germany, 19612000. Agricultural and Forest Meteorology 121(1-2):69-78.

Chmielewski, F.M., and K.P. Götz. 2017. Identification and timing of dormant and ontogenetic phase for sweet cherries in Northeast Germany for modelling purposes. J. Hortic. 4(3):205 doi: 10.4172/23760354.1000205.

De Melo-Abreu, J.P., J.M. Silva, and N.G. Barba. 2005. Flowering date prediction in 'Rocha' pear under mild winter conditions. Acta Hortic. 671:177-182.

Drepper B., A. Gobin, S. Remy, and J. Van Orshoven. 2020. Comparing apple and pear phenology and model performance: what seven decades of observations reveal. Agronomy 10(73):1-21.

Druart N, A. Johansson, B. Kyoko, J. Schrader, A. Sjödin, R. Bhalerao, et al. 2007. Environmental and hormonal regulation of the activity-dormancy cycle in the cambial meristem involves stage-specific modulation of transcriptional and metabolic networks. The Plant Journal 50:557-573.

Fleckinger, J. 1965. Stades repérés es des pomacée s. 45 p. Coll. G. Viennot-Bourgin, J. France.

Gomila T. 2015. Boletin de Madurez № 4. Temporada 2014-2015. Disponible en http://inta.gob.ar/documentos/boletin-demadurez. (Consulta junio de 2016).
Heide, O.M. 1974. Growth and dormancy in Norway spruce (Picea abies). I. Interaction of photoperiod and temperature. Physiol. Plant. 30:1-12.

Heide, O.M. 2008. Interaction of photoperiod and temperature in the control of growth and dormancy of Prunus species. Scientia Horticulturae 115:309-314.

Horvath, D. 2009. Common mechanisms regulate flowering and dormancy. Plant Science 177:523-531.

Kalberer S.R., M. Wisniewski, and R. Arora. 2006. Deacclimation and reacclimation of coldhardy plants: Current understanding and emerging concepts. Plant Science 171:3-16.

Lang, G.A., J.D. Early, G.C. Martin, and R.L. Darnell. 1987. Endodormancy, paradormancy and ecodormancy physiological terminology and classification for dormancy research. HortScience 22:371377.

Luedeling E. 2012. Climate change impacts on Winter chill for temperate fruit and nut production: A review. Scientia Horticulturae 144:218-229.

Luedeling E., L. Guo, J. Dai, C. Leslie, and M. Blanke. 2013. Differential responses of trees to temperature variation during the chilling and forcing phases. Agricultural and Forest Meteorology 181:33-42.

InfoStat. 2009. Programa estadístico Infostat. Versión 2009. Grupo Infostat FCA, Universidad Nacional de Córdoba, Argentina.

Pagter M., J.F Hausman, and R. Arora. 2011. Deacclimation kinetics and carbohydrate changes in stem tissues of Hydrangea in response to an experimental warm spell. Plant Science 180:140-148.

Pascale, D., y E. Damario. 2004. Bioclimatología Agrícola y Agroclimatología. 547 p. Editorial Facultad de Agronomía, Universidad de Buenos Aires, Argentina.

Richardson, E. 1975. Comments on a model for estimating the completion of rest for Red Haven and Elberta peache trees. HortScience 10(6):559-562.

Richardson E., S.D. Seeley, and D.R. Walter. 1974. A model for estimating the completion of rest for 'Red haven' and 'Elberta' peach trees. HortScience 9(4):331-332.

Rodríguez A., y A. Muñoz. 2006. Síntesis agrometeorológica para el período 1990-2004. 38 p. EEA Alto Valle. Boletín de Divulgación Técnica № 53. Ed. INTA, Río Negro, Argentina. 
Rodríguez, A. 2011. Influencia de la temperatura del aire y del raleo sobre el tamaño del fruto de peral (Pyrus comunnis) cv. Williams durante sus distintas fases de desarrollo. 175 p. Tesis de Magíster en Ciencias Agropecuarias. Universidad Nacional de Córdoba, Argentina.

Rodríguez A., E. Sánchez, and A. De la Casa. 2011. Contributions of early season temperatures to Pyrus comunnis 'Bartlett' fruit growth. Acta Hortic. 909(2):657-664.

Russell G.T., B.S. Schilling, N.H. Lowb, G.R. Graya, and L.V. Gustaa. 2014. Cold acclimation deacclimation and re-acclimation of spring canola, winter canola and winter wheat: The role of carbohydrates, cold-induced stress proteins and vernalization. Environmental and Experimental Botany106:156-163.

SENASA. 2017. Anuario estadístico 2017. 154 p. Regional Patagonia Norte. ISSN 25458124. Río Negro, Argentina.

Szalay L., B. Timon, S. Nemeth, J. Papp, and M. Toth. 2010. Hardening and dehardening of peach flower buds. Hortscience 45(5):761765 .
Tanino, K.K., L. Kalcsits, S. Silim, E. Kendall, and G.R. Gray. 2010. Temperature-driven plasticity in growth cessation and dormancy development in deciduous woody plants: a working hypothesis suggesting how molecular and cellular function is affected by temperature during dormancy induction. Plant Mol. Biol. 73:49-65.

Welling A., and E.T. Palva. 2006. Molecular control of cold acclimation in trees. Physiol. Plant. 127:167-181.

Westwood N. 1982. Fruticultura de Zonas Templadas. 461 p. Ed. Mundiprensa, Madrid, España.

Wolfe, D.W., M.D. Schwartz, A.N. Lakso, Y. Otsuki, R.M. Pool, and N.J. Shaulis. 2005. Climate change and shifts in spring phenology of three horticultural woody perennials in northeastern USA. Int. J. Biometeorol. 49:303-309. 